\title{
Trusted Service Evaluation for Mobile Edge Users: Challenges and Reviews
}

\author{
Tingting Shao $\mathbb{D}^{1},{ }^{1}$ Xuan Yang $\mathbb{D},{ }^{2}$ Fan Wang $\mathbb{D},{ }^{3}$ Chao Yan $\mathbb{D},{ }^{3}$ and Ashish Kr. Luhach $\mathbb{D D}^{4}$ \\ ${ }^{1}$ Medical Information Engineering School, Jining Medical University, Jining, China \\ ${ }^{2}$ Weifang Key Laboratory of Blockchain on Agricultural Vegetables, Weifang University of Science and Technology, \\ Weifang, China \\ ${ }^{3}$ School of Computer Science, Qufu Normal University, Jining, China \\ ${ }^{4}$ Department of Electrical and Communications Engineering, The PNG University of Technology, Lae, Papua New Guinea
}

Correspondence should be addressed to Ashish Kr. Luhach; ashish.kumar@pnguot.ac.pg

Received 7 August 2021; Revised 8 September 2021; Accepted 8 December 2021; Published 21 December 2021

Academic Editor: Anirban Chakraborti

Copyright $(92021$ Tingting Shao et al. This is an open access article distributed under the Creative Commons Attribution License, which permits unrestricted use, distribution, and reproduction in any medium, provided the original work is properly cited.

With the increasing growth of web services shared in various mobile edge platforms, it becomes necessary to evaluate all the candidates based on their quality of services to reduce the users' service selection cost. However, the service quality data released by service providers cannot be simply deemed as trusted due to various subjective or objective reasons, which further produce a series of serious trust-aware service evaluation problems, including service quality data sparsity and lack of feedback incentive. In view of this, we summarize the challenging issues existing in the current research field of trusted mobile edge service evaluation. Afterward, we review the current research status of the trusted service evaluation in the mobile edge environment and discuss one of the typical application scenarios based on trusted service evaluation, that is, recommender systems, as well as their diverse categories. We believe this research could be helpful in assisting a mobile edge platform to build a trusted reputation system for various smart applications hosted in the mobile edge platform.

\section{Introduction}

The credibility of network-structure software or web service is vital for building a highly trusted mobile edge computing platform (i.e., edge computing in mobile devices) [1]. Due to the inherent openness and dynamic nature of the mobile edge environment, the running process of web services in a mobile edge platform is often affected by many uncertain factors, which greatly reduces the credibility of service running quality [2]. Therefore, to ensure the normal operation of the mobile edge-based web services or business processes, it is urgent and necessary to study a credibility-guaranteed mechanism for web services. At present, both the academia and industry areas have conducted preliminary explorations and research on the topic of "trusted web services" [3-7] and proposed a series of important research topics, such as trusted selection of web services, trusted combination of collaborative services, and trusted replacement of abnormal services.
Web services selection is the first step for users to invoke Web services and then construct complex mobile edge applications. (Here, the scope of "service" is very wide and comprehensive. Every item that can be provisioned to users could be regarded as a service, e.g., movie, news, blog, commercial products). With the increasing success of service computing technologies in e-Economy [8], e-Science [9], e-Government [10], and other fields, more and more web services are emerging with the same functions in the mobile edge environment. Therefore, when choosing web services, users should not only consider their application requirements in terms of functions but also pay more attention to the nonfunctional quality performance of web services, that is, QoS (quality of service), such as response time, throughput rate. Through objective measurement and evaluation of the QoS natures of each dimension of web services, users can select a web service with the best quality that meets their functional requirements from many 
similarly available candidate web services to participate in their mobile edge-based business execution process.

However, due to the dynamic and unpredictable services running environment and the business competition from the false propaganda and malicious deception, the service QoS data released by service providers are not always truthful [11-14]. This untrusted QoS data will interfere with the normal service selection process of the user and cause users to make the wrong decision and judgment (such as no credible sensor service QoS cause the failure of fire warning). They will destroy the fair and reasonable competition order between service subjects. Therefore, finding more authentic and reliable sources of QoS data to replace untrusted ones published by service providers is crucial for mobile edge users.

In this paper, we focus on the problems and challenges existing in the field of trusted service evaluation in mobile edge computing. Concretely, the remainder of this paper is structured as follows: in Section 2, we summarize the current search challenges and problems in the trusted service evaluation based on historical service quality records. Afterward, in Section 3, we review the current research literature from two aspects: subjective user rating and objective QoS records. In Section 4, we discuss one of the future application patterns of trusted service quality information, that is, recommender systems in mobile edge computing. Finally, in Section 5, we conclude the paper and analyze the improvement directions in future work.

\section{Research Challenges}

In a mobile edge computing environment, users tend to leave a record after invoking the web service, such as subjective user rating (e.g., common rating of " 1 star" to " 5 stars") or objective QoS records (the quality information of the web service in the execution of this invocation, e.g., a web service's response time is 2 seconds). The invocation record more truly reflects the quality of web services in the past; thus, it became one of the most credible bases for measuring the true quality of the web service in the mobile edge environment. At present, academia widely uses the historical invocation record of web services to evaluate the quality of service and select web services to overcome the defect of the unreliability of QoS data published by the service provider in traditional methods [15-18]. However, this method of "web service selection based on historical invocation records in mobile edge environment" still faces many trust problems that need to be solved.

\subsection{Incentives and Preprocessing of Sparse User Ratings.} First of all, due to the lack of an effective incentive mechanism, users are not highly motivated to make ratings after invoking web services. As a result, user ratings of web services are sparse in the mobile edge environment $[19,20]$, which greatly reduces the feasibility and accuracy of evaluating the quality of web services through user ratings of web services. Secondly, to ensure the authenticity of user ratings, malicious ratings of bad users (such as deliberate fraud and malicious collusion between service providers and users) should be identified and punished. However, when a user rating is very sparse, the effect of the traditional malicious rating recognition method based on statistical thinking is not good [21, 22]. Moreover, by doping from the subjective preference of the user, the web service user rating is not the unbiased estimator of the quality of the service, so you need to identify and reversely correct subjective preference in user ratings. However, the traditional, preferred rating recognition method based on a statistical idea requires a large number of known user rating data, which is not suitable for very sparse user ratings.

Generally, we regard the situations where feedback is very sparse as cold-start problems, which often render trusted service evaluation infeasible. As inherent ills in the mobile edge environment, many researchers devote their attention to alleviating cold-start problems for better service selection. Wang et al. [23] incorporate user trust into service evaluation and combine trust relationships with rating records to achieve robust service selection. Wang et al. [24] employed a metalearning embedding ensemble (ML2E) algorithm to perform a more accurate evaluation for new services. However, the above studies do not fundamentally solve the cold-start problems, which need to be further studied in the future.

2.2. Protection and Evaluation of QoS Records. Firstly, the QoS records generated by the user after invoking the web service are also a kind of private data. Therefore, for privacy protection, users are not willing to disclose the monitored QoS record $[25,26]$, which intensifies the sparse QoS record in a mobile edge environment and reduces the feasibility and accuracy of evaluating the quality of web services through the QoS record of web services. Secondly, some QoS natures of web services are not completely independent but correlated with each other $[27,28]$. However, the existing web services evaluation methods (such as the commonly used weighted method) do not consider such attribute correlation, thus reducing the accuracy of the evaluation results of quality of service. Moreover, some web services (such as Mobile edge service) have a longer running cycle (such as running for a week), and their quality of service constantly fluctuates during the running cycle. Therefore, some QoS records for this type of web service are not simply fixed values (quality points) but a quality curve that fluctuates over time [29]. However, the existing web services evaluation methods do not consider this special form of QoS record. Therefore, it is easy to cause the one-sidedness and incompleteness of service quality evaluation, thus reducing the accuracy of service evaluation results.

\subsection{Weight Allocation of Historical Invocation Records.} There are probably multiple historical invocation records for web services that are used more frequently [30] (i.e., multiple user ratings, or multiple QoS records, or a combination of user ratings and QoS records). For the multiple invocation records (such as ratings and rating scores, etc.), the context information (such as invocation time and network 
environment) are probably distinctive. Thus, multiple invocation records for the same web service are not exactly the same for evaluating and forecasting the web service quality. In addition, for different candidate web services, the number of invocation records (i.e., user ratings or QoS records) also varies. To a certain extent, this will also affect the degree of trust of mobile edge users in web service quality. Therefore, treating all invocation records of all candidate web services equally will result in inaccurate web service selection results.

To sum up, in the mobile edge computing environment, with the increasingly intensified competition of web services and the constantly changing service operating environment, QoS data published by service providers may not be true and reliable. Therefore, predicting the future quality of a web service based on its historical invocation record is one of the effective ways to implement trusted service selection. However, due to the sparsity of user ratings, the diversity of QoS records (e.g., diverse privacy requirements, diverse attribute associations, and diverse record forms), and the difference of invocation records, currently "the selection of web services based on historical invocation records in mobile edge environment" still faces many trust problems that need to be solved urgently. Accordingly, we carry out the research of "trusted service selection based on historical invocation records in mobile edge environment" based on the previous achievements. The ultimate research goal is to provide real and reliable service quality reference data for mobile edge users' web services selection and then provide necessary theoretical and technical support for the development and maintenance of highly reliable network software platform when the QoS data released by the service provider is not credible.

\section{Research Review}

At present, the academic community has made an active exploration and research on the topic of "web service selection based on historical invocation records under mobile edge environment", and has gained many phased scientific research achievements. The following summarizes the existing research results from two aspects: subjective user rating and objective QoS records (topic distribution and temporal distribution of mentioned literature are shown in Figures 1 and 2).

3.1. Subjective User Rating. It is summarized from four perspectives (briefly introduced in Table 1): feedback incentive of user rating, identification and punishment of malicious rating, identification and correction of preference rating, and weight allocation of user rating.

3.1.1. Feedback Incentive of User Rating. In order to fundamentally solve the sparsity of user rating, an effective incentive mechanism should be designed to improve the enthusiasm of users for feedback rating. Li et al. [16] calculated the "recommendation trust" of each user, and users with high recommendation trust were given priority to get high attention to encourage users to give positive and credible feedback ratings. According to the previous rating data of users, Yu et al. [32] calculated the credibility of their ratings. Users with high credibility will be given priority to get high-quality service recommendations to improve the enthusiasm of users' ratings. However, the incentive basis of the above incentive mechanism is relatively single, and there is no treatment method for repeated rating. Therefore, the incentive effect is relatively limited, which cannot effectively reduce the sparsity of user rating under the mobile edge environment.

\subsubsection{Identification and Punishment of Malicious Rating.} Malicious rating from bad users will bring great damage and interference to the trust system of the mobile edge platform. Malik et al. identified possible malicious ratings [21] by comparing the rating differences between a single user and a group of users for the same web service. He used a method of analyzing the distribution of a large number of user ratings to find the possible false and malicious rating. Wang et al. detected malicious rating [31] by comparing the normal feedback level and the average step of sampling feedback level. Other malicious rating recognition methods include the recognition method based on pattern analysis [33] and recognition method based on the user registration information [34]. However, the mentioning identification methods aiming at malicious rating mainly depend on a lot of user rating data. Therefore, when a user rating is very sparse, the effect is not satisfactory. In addition, Web services run differently at different times and in different environments, so different users may have different ratings for the same web service. However, the above methods cannot distinguish such normal differences and are easy to "misjudge" the real user ratings.

What is more, in order to encourage users to give real feedback, it is necessary to give a punishment to the malicious ratings from bad users. Witkowski et al. designed a punishment method [35] based on service price. Zhang et al. punished bad users who provided malicious ratings [36] by reducing the trust and attention of bad users. The above punishment methods consider the "benefits" and "risks" of malicious rating by bad users, respectively. However, the punishment basis is relatively single and cannot adapt to the complex web service trust system.

3.1.3. Identification and Correction of Preference Ratings. Identifying the implicit subjective preference in user ratings is conducive to an objective and accurate quality assessment of web services. According to the user's sensitive degree to the quality of service, Li et al. found that users can be divided into tolerant and rigid users. They discussed the rating rules of two types of users: one is positive and the other is negative [16]. Malik and Bouguettaya revealed the positive user rating distribution which is described as to J-shape [21].

3.1.4. Through the Statistical Analysis. In order to minimize the negative impact of the preference rating to evaluate service, we need to do the reverse correction to the preference rating that is identified. Based on user feedback between the ranking 


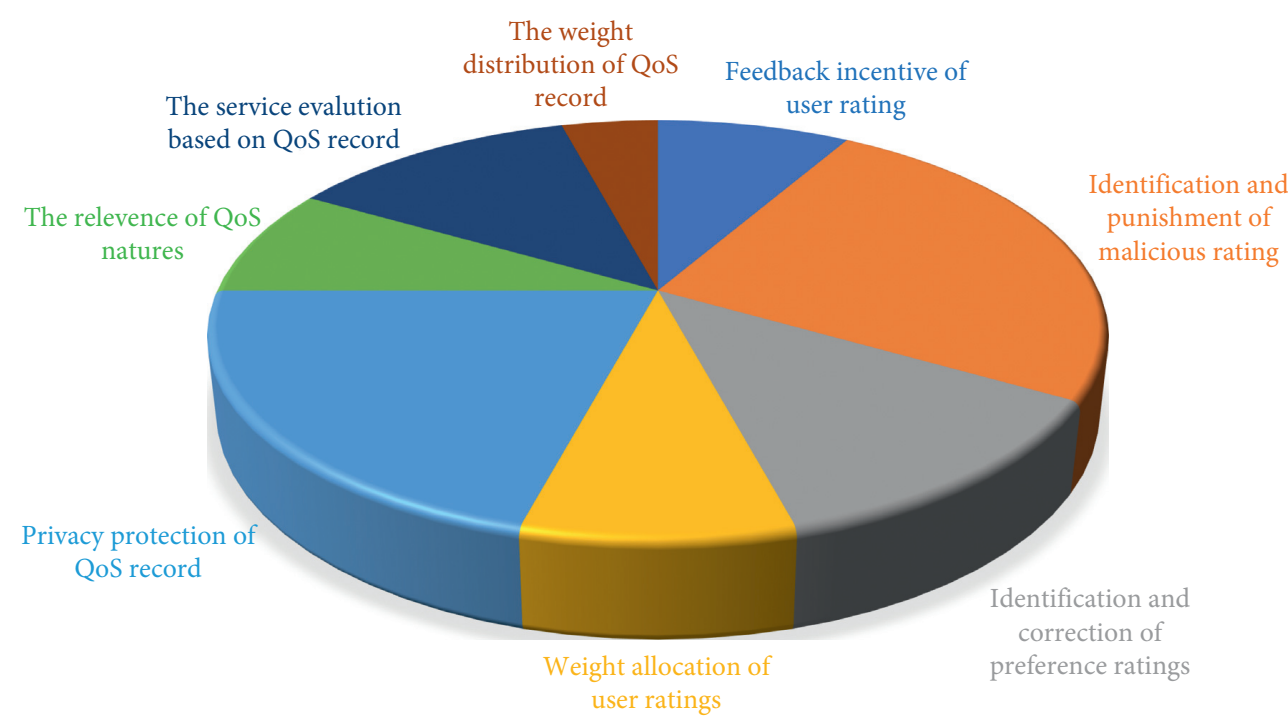

FIGURE 1: Topic distribution of literature about subjective user rating and objective QoS records.

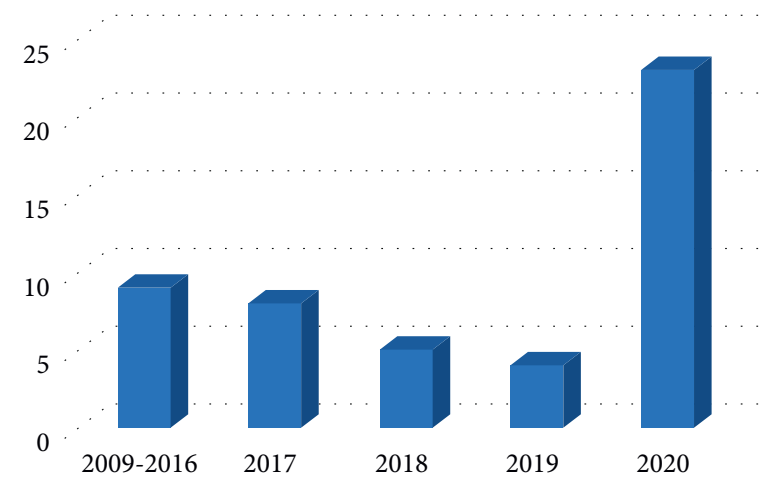

Figure 2: Temporal distribution of recent literature in this study.

TABLE 1: Research protocol of subjective user rating.

\begin{tabular}{|c|c|c|c|c|}
\hline Category & Title & Authors & Year & Publisher \\
\hline \multirow{2}{*}{$\begin{array}{l}\text { Feedback incentive of user } \\
\text { rating }\end{array}$} & $\begin{array}{c}\text { Community-Diversified Influence Maximization in } \\
\text { Social Networks [16] }\end{array}$ & Li et al. & 2020 & Information Systems \\
\hline & $\begin{array}{c}\text { Hybrid Attacks on Model-Based Social Recommender } \\
\text { Systems [32] }\end{array}$ & Yu et al. & 2017 & $\begin{array}{c}\text { Physica A: Statistical } \\
\text { Mechanics \& Its Applications }\end{array}$ \\
\hline \multirow{6}{*}{$\begin{array}{l}\text { Identification and } \\
\text { punishment of malicious } \\
\text { rating }\end{array}$} & $\begin{array}{c}\text { Community-Diversified Influence Maximization in } \\
\text { Social Networks [16] }\end{array}$ & Li et al. & 2020 & Information Systems \\
\hline & $\begin{array}{c}\text { Rater Credibility Assessment in Web Services } \\
\text { Interactions [21] }\end{array}$ & Malik et al. & 2009 & World Wide Web Journal \\
\hline & $\begin{array}{c}\text { Shilling Attack Detection in Recommender Systems via } \\
\text { Selecting Patterns Analysis [33] }\end{array}$ & Li et al. & 2016 & $\begin{array}{l}\text { IEICE Transactions on } \\
\text { Information and System }\end{array}$ \\
\hline & $\begin{array}{l}\text { Identifying Fake Feedback for Effective Trust } \\
\text { Management in Cloud Environments [34] }\end{array}$ & Noor et al. & 2013 & LNCS \\
\hline & $\begin{array}{l}\text { A. Krause. Incentive-Compatible Forecasting } \\
\text { Competitions [35] }\end{array}$ & $\begin{array}{l}\text { Witkowski } \\
\text { et al. }\end{array}$ & 2018 & $\begin{array}{c}\text { Thirty-Second AAAI } \\
\text { Conference on Artificial } \\
\text { Intelligence }\end{array}$ \\
\hline & $\begin{array}{c}\text { Study on the Trust Evaluation Approach Based on } \\
\text { Cloud Model [36] }\end{array}$ & Zhang et al. & 2013 & Chinese Journal of Computers \\
\hline
\end{tabular}


TABLE 1: Continued.

\begin{tabular}{|c|c|c|c|c|}
\hline Category & Title & Authors & Year & Publisher \\
\hline \multirow{3}{*}{$\begin{array}{l}\text { Identification and } \\
\text { correction of preference } \\
\text { ratings }\end{array}$} & $\begin{array}{l}\text { Community-Diversified Influence Maximization in } \\
\text { Social Networks [16] }\end{array}$ & Li et al. & 2020 & Information Systems \\
\hline & $\begin{array}{c}\text { Rater Credibility Assessment in Web Services } \\
\text { Interactions [21] }\end{array}$ & Malik et al. & 2009 & World Wide Web Journal \\
\hline & $\begin{array}{l}\text { Reputation Measurement and Malicious Feedback } \\
\text { Rating Prevention in Web Service Recommendation } \\
\text { System [31] }\end{array}$ & Wang & 2015 & $\begin{array}{l}\text { IEEE Transactions on Services } \\
\text { Computing }\end{array}$ \\
\hline \multirow{2}{*}{$\begin{array}{l}\text { Weight allocation of user } \\
\text { ratings }\end{array}$} & $\begin{array}{c}\text { A Time-Aware Dynamic Service Quality Prediction } \\
\text { Approach for Services [7] }\end{array}$ & Jin et al. & 2020 & $\begin{array}{l}\text { Tsinghua Science and } \\
\text { Technology }\end{array}$ \\
\hline & $\begin{array}{c}\text { An Attention-Based Category-Aware GRU Model for } \\
\text { Next POI Recommendation [15] }\end{array}$ & Liu et al. & 2021 & $\begin{array}{l}\text { International Journal of } \\
\text { Intelligent Systems }\end{array}$ \\
\hline
\end{tabular}

and user context information, Wang et al. calculated the current user's virtual rating and the conflict of virtual rating and the actual rating and then did reverse correction to users with large conflicts [31]. However, the above methods for the identification and correction of preference rating mainly depend on many user rating data. When a user rating is very sparse, the effect will not be beautiful.

3.1.5. Weight Allocation of User Ratings. To accurately assess the true quality of a web service, it is necessary to assign different weights to its user ratings. Jin et al. [7] studied the correlation between the rating time and the rating weight. $\mathrm{Hu}$ et al. used the user rating score size as the design basis of the weighted rating to weaken the negative impact on objective evaluation from the positive user ratings.

In addition, the credibility of user rating will also affect the contribution from user ratings to service quality evaluation. Liu et al. analyzed the correlation between user's credibility and rating weight [15]. However, the above literature focused more on qualitative analysis of various factors affecting rating weight, lack of quantitative theoretical analysis and data support, which results in inability to effectively support the quality assessment of web services based on multirating weighted aggregation.

3.2. Objective QoS Record. It is summarized from the privacy protection of QoS record (briefly introduced in Table 2), QoS relevance, service evaluation based on QoS record, and weight distribution of QoS record.

3.2.1. Privacy Protection of QoS Record. In order to protect the privacy information in QoS records, Razaque et al. [37] incorporated QoS privacy into the contract of SLA (service protection of QoS privacy data through classified privacy.

3.2.2. Contracts. Zhang et al. [41], Wang et al. [43], and Khazbak et al. [44] discussed the possible privacy leakage issues in various domains. For example, the authors introduced the privacy exposure problems and challenges existing in current various sharing economy services, including biking location privacy. In other words, when people are enjoying the convenient services provisioned by biking rental enterprises, they are often confronted with hidden and unsecure privacy issues because the sensors and GPS modules embedded in bikes will monitor and collect the real-time user location information at any time and any place. Moreover, Meng et al. [38] and Wang et al. [39, 40] alleviated the privacy leakage issues of QoS records transmission on the distributed computing platforms. However, all the above literature only studied the privacy protection of QoS records from a higher level and perspective and lacked specific solutions. Therefore, the effect of privacy protection is relatively limited and cannot effectively eliminate users' worries about QoS privacy leakage.

3.2.3. The Relevance of QoS Natures. Part of the QoS natures of Web services is not completely independent but related. Luo and others modeled the relationship among the QoS attributes through the service-related model BSCM [27]. They analyzed the reverse relationship between different QoS attributes. Zhong et al. used the TOPSIS method in multiobjective optimization to make a dimension reduction aimed at multidimensional and associated QoS attributes of a web service [28]. To a certain extent, it has weakened the relevance of QoS natures' negative influence on the service quality evaluation. However, the above literature focused on modeling and qualitative description of QoS natures correlation, lacking quantitative correlation calculation. Many QoS records are needed to support the calculation of attribute correlation, but the scope of application is narrow. In addition, the above literature did not discuss the nonlinear correlation between different QoS natures of web services, which further reduced its application scope.

3.2.4. The Service Evaluation Based on QoS Record. After the QoS records generated after the web service are invocated, trusted web services can be evaluated, selected, and combined. Zhang et al. used the historical records of web services to predict the future preferences of users and make appropriate recommendation decisions [41]. Zhong et al. [28] used the QoS records of web services to select the best web service through multiquality evaluation. Malik et al. [21] determined the quality of the web service credibility by comparing the web service QoS record with its promise of SLA quality level. However, the above literature all assumed that the web service QoS record is a 
TABLE 2: Research protocol of objective user rating.

\begin{tabular}{|c|c|c|c|c|}
\hline Category & Title & Authors & $\begin{array}{l}\text { Publication } \\
\text { year }\end{array}$ & Publisher \\
\hline \multirow{5}{*}{$\begin{array}{l}\text { Privacy protection of } \\
\text { QoS record }\end{array}$} & $\begin{array}{l}\text { Privacy Preserving Model: A New Scheme for } \\
\text { Auditing Cloud Stakeholders [37] }\end{array}$ & $\begin{array}{l}\text { Razaque } \\
\text { et al. }\end{array}$ & 2017 & Journal of Cloud Computing \\
\hline & $\begin{array}{c}\text { Amplified LSH-Based Recommender Systems with } \\
\text { Privacy Protection [25] }\end{array}$ & Chi et al. & 2020 & $\begin{array}{c}\text { Concurrency and } \\
\text { Computation: Practice and } \\
\text { Experience }\end{array}$ \\
\hline & $\begin{array}{c}\text { Security-Aware Dynamic Scheduling for Real-Time } \\
\text { Optimization in Cloud-Based Industrial } \\
\text { Applications [38] }\end{array}$ & $\begin{array}{l}\text { Meng } \\
\text { et al. }\end{array}$ & 2021 & $\begin{array}{l}\text { IEEE Transactions on } \\
\text { Industrial Informatics }\end{array}$ \\
\hline & $\begin{array}{c}\text { An Optimization and Auction Based Incentive } \\
\text { Mechanism to Maximize Social Welfare for Mobile } \\
\text { Crowdsourcing [39] }\end{array}$ & $\begin{array}{l}\text { Wang } \\
\text { et al. }\end{array}$ & 2019 & $\begin{array}{l}\text { IEEE Transactions on } \\
\text { Computational Social Systems }\end{array}$ \\
\hline & $\begin{array}{l}\text { A Novel Hybrid Method to Analyze Security } \\
\text { Vulnerabilities in Android Applications [40] }\end{array}$ & Tang et al. & 2020 & $\begin{array}{l}\text { Tsinghua Science and } \\
\text { Technology }\end{array}$ \\
\hline \multirow{2}{*}{$\begin{array}{l}\text { The relevance of QoS } \\
\text { natures }\end{array}$} & $\begin{array}{l}\text { Business Correlation-Aware Modeling and Services } \\
\text { Selection in Business Service Ecosystem [27] }\end{array}$ & Luo et al. & 2013 & $\begin{array}{l}\text { International Journal of } \\
\text { Computer Integrated } \\
\text { Manufacturing }\end{array}$ \\
\hline & $\begin{array}{c}\text { Multi-Dimensional Quality-Driven Service } \\
\text { Recommendation with Privacy-Preservation in } \\
\text { Mobile Edge Environment [28] }\end{array}$ & $\begin{array}{l}\text { Zhong } \\
\text { et al. }\end{array}$ & 2020 & Computer Communications \\
\hline \multirow{3}{*}{$\begin{array}{l}\text { The service evaluation } \\
\text { based on QoS record }\end{array}$} & $\begin{array}{c}\text { Deep Sequential Model for Anchor } \\
\text { Recommendation on Live Streaming Platforms [41] }\end{array}$ & $\begin{array}{l}\text { Zhang } \\
\text { et al. }\end{array}$ & 2021 & Big Data Mining and Analytics \\
\hline & $\begin{array}{c}\text { Multi-Dimensional Quality-Driven Service } \\
\text { Recommendation with Privacy-Preservation in } \\
\text { Mobile Edge Environment [28] }\end{array}$ & $\begin{array}{l}\text { Zhong } \\
\text { et al. }\end{array}$ & 2020 & Computer Communications \\
\hline & $\begin{array}{c}\text { Rater Credibility Assessment in Web Services } \\
\text { Interactions [21] }\end{array}$ & $\begin{array}{l}\text { Malik } \\
\text { et al. }\end{array}$ & 2009 & World Wide Web Journal \\
\hline $\begin{array}{l}\text { The weight } \\
\text { distribution of QoS } \\
\text { record }\end{array}$ & $\begin{array}{c}\text { How Textual Quality of Online Reviews Affect } \\
\text { Classification Performance: A Case of Deep } \\
\text { Learning Sentiment Analysis [42] }\end{array}$ & Li et al. & 2020 & $\begin{array}{l}\text { Neural Computing and } \\
\text { Applications }\end{array}$ \\
\hline
\end{tabular}

simple fixed value (i.e., the quality point), the diversity of QoS record is not considered under the mobile edge environment (i.e., quality, quality curve) and their integration problem. Therefore, it is likely to cause the partial and incomplete problem of service quality evaluation, thus reducing the accuracy of the service evaluation results.

3.2.5. The Weight Distribution of QoS Record. In order to evaluate the real quality of a web service more accurately, different weights should be assigned to each QoS record of a web service. Li et al. analyzed the correlation between QoS record time and the weight [42]. At present, there are few studies on this aspect, which cannot effectively support web service quality assessment based on the weighted aggregation of multi-QoS records. Other similar work can be found in $[10,45-47]$, where the multiple-dimensional weighting issue is studied in various ways.

\section{Future Directions: Service Quality- Driven Recommendation}

Through utilizing the existing and known web service quality data (including objective QoS records and subjective user ratings), we can perform personalized service recommendation for prospective users. This section discusses one of the research directions using service quality information: recommender systems. Generally, the research field can be divided into the following six categories.

4.1. Content-Based Recommender Systems. Service content is mainly about the details of "what" the service executed by users involves or discusses. For example, a user watches a movie named "Roman Holiday" whose actress is Audrey Hepburn and the movie genre is Love. Then, according to content-based recommendation theory, in the future, the user would be recommended the movies whose actress is Audrey Hepburn and whose genre is Love. In other words, content-based recommendation only considers the information contained in the content of the services ever executed by users, without involving other people.

The advantages of content-based recommendation theory are that it does not need to consider the information of other people. Instead, it only needs to know about the historically executed service information of a target user himself. Therefore, if the historical service execution data are rare or sparse, a content-based recommendation is a promising solution to alleviate the sparse data or cold-start recommendation issues.

4.2. Knowledge-Based Recommender Systems. Knowledge is often a key information source in various computing-intensive smart or intelligent applications, such as gambling, chess, and 
mathematical reasoning. Likewise, in recommender systems, knowledge also plays an essential role in outputting a group of high-quality recommended items. For example, if TV says it will be rainy today, then you would be recommended to take an umbrella when you intend to go outside, as there is an obvious knowledge between rain and an umbrella. Besides the obvious knowledge mentioned above, there is also various knowledge that is hidden and implicit. For example, if Alice took a taxi to the hospital at 2:00 a.m., there is an implicit knowledge that Alice was very sick. Such implicit knowledge also contributes much to improving user satisfaction when obvious knowledge is absent from the decision-making process.

Typically, a knowledge graph (KG) provides a promising way for service recommendation and draws attention to researchers in the field of knowledge-based recommender systems. Zhang et al. [48] modeled the collaborative filtering problem as a knowledge graph for link prediction and recommendation. However, this research does not consider privacy protection. In light of this, Yu et al. [49] employed the Laplacian noise to optimize recommendation process based on KG. However, the above literature only leveraged a single relationship to construct the KG framework, which is difficult to cover multiple relationships in practice. To address this issue, Shi et al. [50] put forward a multidimensional knowledge graph framework to recommend personalized learning paths for E-learners. In summary, the advantage of knowledge-based recommendation is that it is precise and accurate as the knowledge can capture the users' preferences well, while the disadvantage is that the knowledge is often not easy to capture as sometimes it is hidden in data and implicit enough.

\subsection{Association Rule-Based Recommender Systems.} Association rule is the valuable information contained in the correlation among different data dimensions. Association rule implies the hidden knowledge extracted from big data and can be used to make directional information reduction. For example, we can infer or predict the future pork price in a certain time period through analyzing the user reviews, blogging, and sum-ups recorded on the Web because there is an association rule between the pork price and the web information. This way, through association rules, we can reduce the heavy burden on frequent economic statistics activities.

The advantages of the association rules-based recommendations are that the association rules are mined from big data and can accurately reflect the correlation relationships among different things involved. The disadvantages are that association rules are often difficult to mine and obtain, especially when the available data for mining are sparse.

4.4. Collaborative Recommender Systems. The collaborative recommendation is one of the most understandable recommendation manners and has been widely employed in various industrial fields. The basic idea of collaborative recommendation is through similarity calculation. For example, if Alice is a similar friend to Tom, then we can recommend the things liked by Alice to Tom, vice versa, which is the basic idea of user collaboration-based recommender systems. Another example is that if a user likes Coca-Cola, then we can recommend Pepsi Cola to them as Coca-Cola and Pepsi Cola are similar drinks to some extent, which is the basic idea of item collaboration-based recommender systems. Thus, through calculating various similarity values, we can make corresponding recommendations to users.

The advantage of this recommendation way is that it is easy to interpret and can be applied to various fields. The disadvantage is that it fails in delivering a quick response as the similarity calculation is often computation-intensive and time-consuming. Therefore, it is not very suitable for the big data application environment where quick responses are needed.

\subsection{Demography-Based Recommender Systems.} Demography contains a variety of useful information that depicts users' profiles, such as the users' age, salary, sex, education degree, and working positions. These pieces of individual information collectively constitute the personalized profile of a user and, therefore, can predict the user preferences well. For example, a professor in a university is apt to buy the tools associated with education; a rich man often buys some luxury goods.

The rationale behind demography-based recommender systems is easy to interpret, which is the main advantage of this recommendation way. On the contrary, the disadvantage is that it cannot capture the user preferences accurately and dynamically, as user preferences are often variable with time and not fixed at all. Therefore, it is often inappropriate to use only the demography information for a successful recommender system.

4.6. Hybrid Recommender Systems. If a recommender system combines more than one recommendation technique, it is called a hybrid recommender system. Generally, any successful recommender system is hybrid, such as Amazon, Alibaba, and eBay, as hybrid recommender systems can integrate the advantages of all the involved recommendation manners. As a result, hybrid recommender systems can bring better user experiences and satisfaction.

\section{Conclusions}

Trusted service evaluation based on historical service quality records is crucial for a mobile edge platform to build a dependable service reputation system. However, due to dynamic service execution context and malicious commercial competition, the QoS data released by service providers are often not trusted, especially for a newcomer of a mobile edge platform. Given this drawback, we review the current literature of the trusted service evaluation in mobile edge computing and analyze the challenging issues existing in the field. As a promising extension, we discuss one of the killer applications of trusted service evaluation: recommender systems. We believe this research could be helpful in assisting a mobile edge platform build a trusted reputation 
system so as to assist the successful deployment of various service-related smart applications.

In the mobile edge computing environment, data collaboration among different mobile devices or edge terminals is inevitable [51-59]. Therefore, how to secure user privacy (including privacy measurement) while guaranteeing other conflicting performances in service evaluation is an open research issue that calls for intensive study. In addition, computational overload is normal in the big data environment [60-66]. Therefore, how to effectively offload the heavy computational tasks or jobs in peak time still requires challenging efforts.

\section{Data Availability}

This study is a review article, so no data are available.

\section{Conflicts of Interest}

The authors declare no conflicts of interest regarding the submission, and the manuscript has not been submitted to other journals or conferences for consideration.

\section{References}

[1] M. R. Khosravi and S. Samadi, "BL-ALM: a Blind Scalable edge-Guided Reconstruction filter for smart environmental monitoring through green IoMT-UAV networks," IEEE Transactions on Green Communications and Networking, vol. 5, no. 2, pp. 727-736, 2021.

[2] Z. Cai, Z. He, X. Guan, and Y. Li, "Collective data-Sanitization for Preventing sensitive information inference Attacks in social networks," IEEE Transactions on Dependable and Secure Computing, vol. 15, no. 4, pp. 577-590, 2018.

[3] M. R. Khosravi and S. Samadi, "Reliable data aggregation in Internet of ViSAR Vehicles using Chained Dual-Phase Adaptive Interpolation and data embedding," IEEE Internet of Things Journal, vol. 7, no. 4, pp. 2603-2610, 2020.

[4] W. Zhang, Z. Li, and X. Chen, "Quality-aware user Recruitment based on Federated learning in mobile Crowd sensing," Tsinghua Science and Technology, vol. 26, no. 6, pp. 869-877, 2021.

[5] Z. Xue and H. Wang, "Effective Density-based Clustering algorithms for incomplete data," Big Data Mining and Analytics, vol. 4, no. 3, pp. 183-194, 2021.

[6] X. Zheng and Z. Cai, "Privacy-preserved data sharing towards multiple Parties in industrial IoTs," IEEE Journal on Selected Areas in Communications, vol. 38, no. 5, pp. 968-979, 2020.

[7] Y. Jin, W. Guo, and Y. Zhang, "A time-aware dynamic service quality prediction Approach for services," Tsinghua Science and Technology, vol. 25, no. 2, pp. 227-238, 2020.

[8] E. Jiang, L. Wang, and J. Wang, "Decomposition-based multiobjective optimization for Energy-aware distributed hybrid Flow Shop Scheduling with Multiprocessor tasks," Tsinghua Science and Technology, vol. 26, no. 5, pp. 646-663, 2021.

[9] J. Mabrouki, M. Azrour, D. Dhiba, Y. Farhaoui, and S. E. Hajjaji, "IoT-based data Logger for Weather monitoring using Arduino-based Wireless sensor networks with Remote Graphical application and Alerts," Big Data Mining and Analytics, vol. 4, no. 1, pp. 25-32, 2021.

[10] R. Kumari, S. Kumar, R. C. Poonia et al., "Analysis and predictions of Spread, Recovery, and Death caused by
COVID-19 in India," Big Data Mining and Analytics, vol. 4, no. 2, pp. 65-75, 2021.

[11] F. Wang, H. Zhu, G. Srivastava, S. Li, M. R. Khosravi, and L. Qi, "Robust collaborative filtering recommendation with user-item-trust records," IEEE Transactions on Computational Social Systems, pp. 1-11, 2021.

[12] Y. Xu, C. Zhang, G. Wang, Z. Qin, and Q. Zeng, “A Blockchain-enabled Deduplicatable data Auditing mechanism for network Storage services," IEEE Transactions on Emerging Topics in Computing, vol. 9, no. 3, pp. 1421-1432, 2021.

[13] L. Zhang, J. Liu, F. Shang, G. Li, J. Zhao, and Y. Zhang, "Robust Segmentation method for Noisy Images based on an Unsupervised Denosing filter," Tsinghua Science and Technology, vol. 26, no. 5, pp. 736-748, 2021.

[14] Y. Xu, Ju Ren, Y. Zhang, C. Zhang, Bo Shen, and Y. Zhang, "Blockchain Empowered Arbitrable data Auditing Scheme for network Storage as a service," IEEE Transactions on Services Computing, vol. 13, no. 2, pp. 289-300, 2020.

[15] Y. Liu, A. Pei, F. Wang et al., "An attention-based Categoryaware GRU model for Next POI recommendation," International Journal of Intelligent Systems, vol. 36, 2021.

[16] J. Li, T. Cai, K. Deng, X. Wang, T. Sellis, and F. Xia, "Community-diversified influence maximization in social networks," Information Systems, vol. 92, pp. 1-12, 2020.

[17] Q. Liu, G. Wang, X. Liu, T. Peng, and J. Wu, "Achieving reliable and secure services in cloud computing environments," Computers \& Electrical Engineering, vol. 59, pp. 153-164, 2017.

[18] X. Liu, Q. Liu, T. Peng, and J. Wu, "Dynamic access policy in cloud-based personal health record (PHR) systems," Information Sciences, vol. 379, no. 2, pp. 62-81, 2017.

[19] P. Nitu, J. Coelho, and P. Madiraju, "Improvising personalized Travel recommendation system with recency effects," Big Data Mining and Analytics, vol. 4, no. 3, pp. 139-154, 2021.

[20] L. Qi, C. Hu, X. Zhang et al., "Privacy-aware data Fusion and prediction with spatial-temporal context for smart City industrial environment," IEEE Transactions on Industrial Informatics, vol. 17, no. 6, 2020.

[21] Z. Malik and A. Bouguettaya, "Rater credibility assessment in web services Interactions," World Wide Web Journal, vol. 12, no. 1, pp. 3-25, 2009.

[22] T. Cai, J. Li, S. M. Ajmal, R. Li, and J. X. Yu, "Target-aware holistic influence maximization in spatial social networks," IEEE Transactions on Knowledge and Data Engineering, 2020.

[23] F. Wang, W. Zhong, X. Xu, W. Rafique, Z. Zhou, and L. Qi, "Privacy-aware cold-start recommendation based on collaborative filtering and Enhanced trust," in Proceedings of the IEEE International Conference on Data Science and Advanced Analytics (DSAA), Sydney, NSW, Australia, October 2020.

[24] H. Wang and Y. Zhao, "ML2E: meta-learning embedding ensemble for cold-start recommendation," IEEE Access, vol. 8, Article ID 165757, 2020.

[25] X. Chi, C. Yan, H. Wang, W. Rafique, and L. Qi, "Amplified lsh-based recommender systems with privacy protection," Concurrency and Computation: Practice and Experience, 2020.

[26] M. S. Mahmud, J. Z. Huang, S. Salloum, T. Z. Emara, and K. Sadatdiynov, "A Survey of data Partitioning and sampling methods to support big data analysis," Big Data Mining and Analytics, vol. 3, no. 2, pp. 85-101, 2020.

[27] Y. Luo, Y. Fan, and H. Wang, "Business correlation-aware modeling and services selection in business service Ecosystem," International Journal of Computer Integrated Manufacturing, vol. 26, no. 8, pp. 772-785, 2013. 
[28] W. Zhong, X. Yin, X. Zhang et al., "Multi-dimensional qualityDriven service recommendation with privacy-Preservation in mobile edge environment," Computer Communications, vol. 157, pp. 116-123, 2020.

[29] L. Qi, X. Wang, X. Xu, W. Dou, and S. Li, "Privacy-aware Cross-platform service recommendation based on Enhanced Locality-sensitive Hashing," IEEE Transactions on Network Science and Engineering, vol. 8, 2020.

[30] J. Hu, Yi Pan, T. Li, and Y. Yang, "TW-Co-MFC: two-level weighted collaborative Fuzzy Clustering based on Maximum Entropy for multi-view data," Tsinghua Science and Technology, vol. 26, no. 2, pp. 185-198, 2021.

[31] S. Wang, Z. Zheng, Z. Wu, R. Lyu, and F. Yang, "Reputation measurement and malicious feedback rating Prevention in web service recommendation system," IEEE Transactions on Services Computing, vol. 8, pp. 755-767, 2015.

[32] J. Yu, M. Gao, W. Rong, W. Li, Q. Xiong, and J. Wen, "Hybrid Attacks on model-based social recommender systems," Physica A: Statistical Mechanics and its Applications, vol. 483, pp. 171-181, 2017.

[33] W. Li, M. Gao, H. Li, J. Zeng, Q. Xiong, and S. Hirokawa, "Shilling Attack detection in recommender systems via selecting patterns analysis," IEICE Transactions on Information and System, vol. E99-D, no. 10, pp. 2600-2611, 2016.

[34] T. H. Noor, Q. Z. Sheng, A. Abdullah, J. Law, H. Anne, and H. Ngu, "Identifying Fake feedback for effective trust Management in cloud environments," Lecture Notes in Computer Science, Springer, vol. 7759, pp. 47-58, , Berlin, Heidelberg, 2013.

[35] J. Witkowski, R. Freeman, J. W. Vaughan, and D. M. Pennock, "Andreas Krause. Incentive-Compatible Forecasting Competitions," in Proceedings of the Thirty-Second Association for the Advancement of Artificial Intelligence Conference on Artificial Intelligence, New Orleans, Louisiana, USA, February 2018.

[36] S. Zhang and C. Xu, "Study on the trust evaluation Approach based on cloud model," Chinese Journal of Computers, vol. 36, no. 2, pp. 422-431, 2013.

[37] A. Razaque and S. S. Rizvi, "Privacy preserving model: a new scheme for auditing cloud stakeholders," Journal of Cloud Computing, 2017.

[38] S. Meng, W. Huang, X. Yin et al., "Security-aware dynamic Scheduling for real-time optimization in cloud-based industrial applications," IEEE Transactions on Industrial Informatics, vol. 17, no. 6, pp. 4219-4228, 2021.

[39] Y. Wang, Z. Cai, Z.-H. Zhan, Y. Gong, and X. Tong, “An optimization and Auction based incentive mechanism to Maximize social Welfare for mobile Crowdsourcing," IEEE Transactions on Computational Social Systems, vol. 6, no. 3, pp. 414-429, 2019.

[40] J. Tang, R. Li, K. Wang, X. Gu, and Z. Xu, "A Novel hybrid method to analyze Security Vulnerabilities in Android applications," Tsinghua Science and Technology, vol. 25, no. 5, pp. 589-603, 2020.

[41] S. Zhang, H. Liu, J. He, S. Han, and X. Du, "Deep Sequential model for Anchor recommendation on live Streaming platforms," Big Data Mining and Analytics, vol. 4, no. 3, pp. 173-182, 2021.

[42] Li Lin, T.-T. Goh, and D. Jin, "How Textual quality of online reviews affect Classification performance: a Case of Deep learning Sentiment analysis," Neural Computing \& Applications, vol. 32, pp. 4387-4415, 2020.

[43] Y. Wang, Z. Cai, X. Tong, Y. Gao, and G. Yin, "Truthful incentive mechanism with location privacy-Preserving for mobile Crowdsourcing systems," Computer Network, vol. 135, pp. 32-43, 2018.

[44] Y. Khazbak, J. Fan, S. Zhu, and G. Cao, "Preserving personalized location privacy in Ride-Hailing service," Tsinghua Science and Technology, vol. 25, no. 6, pp. 743-757, 2020.

[45] M. R. Khosravi and S. Samadi, "Data Compression in Visar sensor networks using non-linear adaptive weighting," EURASIP Journal on Wireless Communications and Networking, vol. 264, 2019.

[46] Y. Wang, Z. Cai, Z.-H. Zhan, B. Zhao, X. Tong, and L. Qi, "Walrasian Equilibrium-based multi-objective optimization for task allocation in mobile Crowdsourcing," IEEE Transactions on Computational Social Systems, 2020.

[47] M. R. Khosravi, "ACI: A bar Chart Index for non-linear Visualization of data embedding and aggregation Capacity in IoMT multi-source Compression," Wireless Networks, 2021.

[48] Y. Zhang, J. Wang, and J. Luo, "Knowledge graph embedding based collaborative filtering," IEEE Access, vol. 8, Article ID 134553, 2020.

[49] B. Yu, C. Zhou, C. Zhang, G. Wang, and Y. Fan, "A privacyPreserving multi-Task framework for knowledge graph Enhanced recommendation," IEEE Access, vol. 8, Article ID 115717, 2020.

[50] D. Shi, T. Wang, H. Xing, and H. Xu, "A learning path recommendation model based on A multidimensional knowledge graph framework for E-learning," KnowledgeBased Systems, vol. 195, Article ID 105618, 2020.

[51] Y. Li, H. Ma, L. Wang, S. Mao, and G. Wang, "Optimized content Caching and user association for edge computing in Densely deployed Heterogeneous networks," IEEE Transactions on Mobile Computing, 2020.

[52] M. R. Khosravi, H. Basri, H. Rostami, and S. Samadi, "Distributed random Cooperation for VBF-based Routing in highSpeed Dense Underwater Acoustic sensor networks," The Journal of Supercomputing, vol. 74, no. 11, pp. 6184-6200, 2018.

[53] Y. Li, S. Xia, M. Zheng, B. Cao, and Q. Liu, "Lyapunov optimization based Trade-Off policy for mobile cloud offloading in Heterogeneous Wireless networks," IEEE Transactions on Cloud Computing, 2019.

[54] C. Hu, W. Fan, E. Zen et al., "A Digital Twin-Assisted realtime Traffic data prediction method for 5G-enabled Internet of Vehicles," IEEE Transactions on Industrial Informatics, 2021.

[55] X. Zhou, L. Yue, and W. Liang, "CNN-RNN based intelligent recommendation for online Medical Pre-Diagnosis support," IEEE/ACM Transactions on Computational Biology and Bioinformatics, vol. 18, no. 3, pp. 912-921, 2020.

[56] M. R. Khosravi and S. Samadi, "Efficient payload communications for iot-enabled visar vehicles using discrete cosine transform-based quasi-sparse bit injection," EURASIP Journal on Wireless Communications and Networking, 2019.

[57] J. Cai, Z. Huang, L. Liao, J. Luo, and W.-Xi Liu, "APPM: Adaptive Parallel processing mechanism for service function Chains," IEEE Transactions on Network and Service Management, vol. 18, no. 2, pp. 1540-1555, 2021.

[58] J. Luo, J. Li, L. Jiao, and J. Cai, "On the effective Parallelization and Near-Optimal deployment of service function Chains," IEEE Transactions on Parallel and Distributed Systems, vol. 32, no. 5, pp. 1238-1255, 2021.

[59] Y. Li, Z. Zhang, S. Xia, and H. H. Chen, "A Load-Balanced Reembedding Scheme for Wireless network Virtualization," IEEE Transactions on Vehicular Technology, vol. 70, 2021. 
[60] X. Hu, S. Peng, B. Guo, and P. Xu, "Accurate AM-FM Signal Demodulation and Separation using Nonparametric Regularization method," Signal Processing, vol. 186, pp. 108-131, 2021.

[61] X. Zhou, W. Liang, S. Shimizu, J. Ma, and Q. Jin, "Siamese neural network based few-shot learning for anomaly detection in industrial cyber-physical systems," IEEE Transactions on Industrial Informatics, vol. 17, 2020.

[62] X. Xu, Z. Fang, J. Zhang et al., "Edge content Caching with Deep Spatiotemporal Residual network for IoV in smart City," ACM Transactions on Sensor Networks, vol. 17, no. 3, pp. 1-33, 2021.

[63] X. Hu, S. Peng, and W. L. Hwang, "EMD Revisited: a new understanding of the Envelope and Resolving the ModeMixing problem in AM-FM Signals," IEEE Transactions on Signal Processing, vol. 60, no. 3, pp. 1075-1086, 2012.

[64] X. Zhou, X. Xu, W. Liang et al., "Intelligent Small Object detection based on Digital Twinning for smart Manufacturing in industrial CPS," IEEE Transactions on Industrial Informatics, 2021.

[65] Y. Li, S. Xia, Q. Yang, G. Wang, and W. Zhang, "Lifetimepriority-driven Resource allocation for WNV-based Internet of things," IEEE Internet of Things Journal, vol. 8, no. 6, pp. 4514-4525, 2021.

[66] X. Zhou, W. Liang, K. I. K. Wang, H. Wang, T. Y. Laurence, and Q. Jin, "Deep learning Enhanced Human activity recognition for Internet of Healthcare things," IEEE Internet of Things Journal, vol. 7, no. 7, pp. 6429-6438, 2020. 